\title{
Design Error Diagnosis in Digital Circuits without Error Model
}

\author{
Raimund Ubar', Dominique Borrione \\ TIMA Laboratory, Grenoble
}

raiub@pld.ttu.ee,Dominique.Borrione@imag.fr

Key words: combinational circuits, design errors, stuck-at fault simulation, diagnostic analysis, redesign based error correcting

\begin{abstract}
We describe a new method for design error diagnosis in digital circuits that doesn't use any error model. For representing the information about erroneous signal paths in the circuit, a stuck-at fault model is used. This allows to adopt the methods and tools of fault diagnosis used in hardware testing for the use in design error diagnosis. A diagnostic specific pre-analysis of the circuit based on stuck-at fault model extracts iteratively subcircuits suspected to be erroneous. Contrary to other published works, here the necessary re-synthesis of the extracted subcircuit need not be applied to the whole function of an internal signal in terms of primary inputs, but may stop at arbitrary nodes inside the circuit. As the subcircuits to be redesigned are kept as small as possible, the redesign procedure is simple and fast. The search for subcircuits to be rectified is carried out by a systematic procedure. The procedure is guided by a heuristic priority function derived from the results of diagnostic pre-analysis. Experimental data show the high speed of diagnostic preanalysis.
\end{abstract}

${ }^{1}$ On the leave from Tallinn Technical University, Estonia

The original version of this chapter was revised: The copyright line was incorrect. This has been corrected. The Erratum to this chapter is available at DOI: 10.1007/978-0-387-35498-9_57 


\section{INTRODUCTION}

Design error diagnosis plays an essential role in providing correct VLSI products. Despite the use of CAD tools to produce correct by construction circuits, experience shows that a phase of design correction is necessary [1]. Designs made by CAD tools are manually modified to improve some aspects such as performance or area overhead. During this phase, errors are likely to be inserted. Automatic error diagnosis saves a lot of design debugging time. Existing logic rectification approaches can be classified: error-model based [2-5], structure based [6-8], and re-synthesis based approaches [9-11].

In error model based approaches, the implementation is rectified by matching the error with an error type in the model. The method is restricted because it may fail in error cases not covered by the model. The multiple error case has not been investigated at all because of complexity.

In [6], a structural approach was proposed for engineering change [12]. In order to re-use the engineering effort spent on the old implementation, logic rectification is performed to realize the new specification by modifying the old design. Verification techniques are used to narrow down the potential error region. Then a heuristic called back-substitution is employed in hopes of fixing the error incrementally. This approach requires that a structural correspondence between the specification and the implementation be provided. If this requirement is not fulfilled the method cannot be used.

Re-synthesis approaches are more general; they rely on the symbolic error-diagnosis techniques to find an internal signal in the implementation that satisfies the single fix condition, i.e. the condition of fixing the entire implementation by changing the function of an internal signal. Once such a signal is found, a new function is realized to replace the old function of this signal to fix the error. In the worst case, it may completely re-synthesize every primary output function. The major drawback is that it cannot handle larger designs, because it uses Binary Decision Diagrams (BDD) [13].

In this paper, the re-synthesis approach is applied not to the whole function of an internal signal given in terms of input signals, but to internal subfunctions for smaller subcircuits. By diagnostic preanalysis, a subcircuit suspected to be erroneous is extracted and redesigned to match the verification results. If the redesign does not solve the problem (cannot correct the circuit), the initial extracted subcircuit is extended either towards the inputs or towards the outputs, and the redesign procedure is repeated. As the subcircuits to be redesigned are as small as possible, the redesign procedure is simple and fast. The size of the subcircuit depends substantially on the quality of diagnostic pre-analysis. In the worst case of many design errors, or if the errors are spread all over the circuit it may be needed to redesign all the circuit, as in the case of known methods $[9,11]$. 


\section{PRELIMINARY}

Consider a circuit specification, and implementation, both at the Boolean level. The specification output is given by a set of variables $W=\left\{w_{1}\right\}$, and the implementation output is given by a set of variables $Y=\left\{y_{j}\right\}$. Let $X=$ $\left\{x_{k}\right\}$ be the set of input variables. The implementation is a network of components (gates), and $Z$ is the set of connections (edges) between components, labelled by signal variables. Let $S$ be the set of all variables in the implementation $S=Y-Z-X$. The components are described by Boolean functions $s=f\left(s_{1}, s_{2}, \ldots, \overline{s_{h}}\right)$ where $s_{-} Y_{-} Z, s_{l}-Z{ }_{-} X$. If $s_{k}$ is a fanout variable then all the branches of the fanout are denoted by the second index: $s_{k, 1}, s_{k, 2}, \ldots, s_{k, p}$, where $p$ is the number of branches. Denote the subset of variables in $Z$ which represent the branches of fanout signals as $Z^{B}$.

Example: In Fig. 1 a combinational circuit is represented with $X=\left\{s_{1}, s_{2}\right.$, $\left.s_{3}, s_{4}, s_{5}, s_{6}, s_{7}\right\}, \quad Z=\left\{s_{3,1}, s_{3,2}, s_{8,}, s_{8,1}, s_{8,2}, s_{9,} s_{10}, s_{11}, s_{12}, s_{13}, s_{14}\right\}, \quad Z^{B}=\left\{s_{3,1}, s_{3,2}, s_{8,1}, s_{8,2}\right\}$, and $Y=\left\{s_{15}\right\}$. We assume that there exists a double design error in the circuit: $A N D_{12}$ should be XOR gate, and $O R_{14}$ should be AND gate.

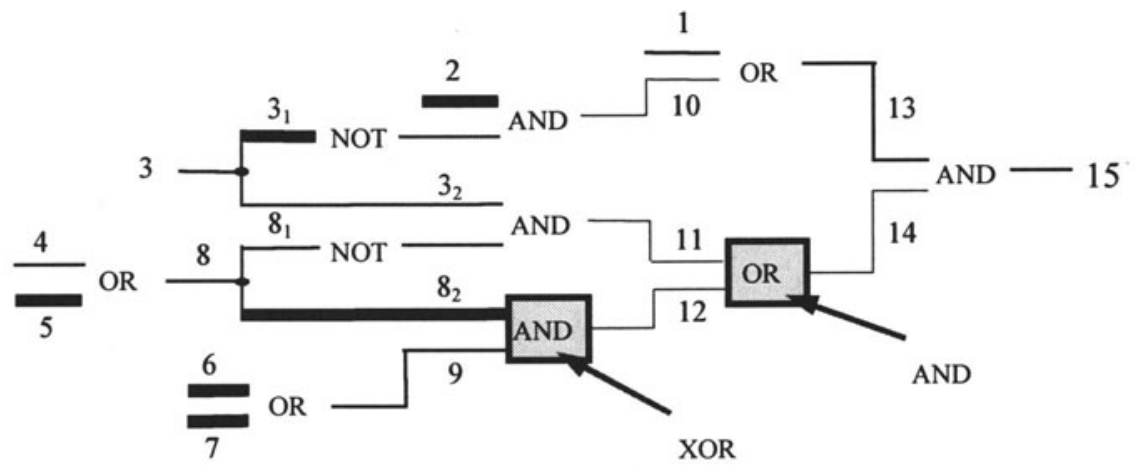

Figure 1. Combinational circuit with two design errors

Definition 1. The cone $C\left(s_{k}\right), s_{k}-S$, is the subset of all variables $s \quad S$ from which there exists a path from $\bar{s}$ to $s_{k}$ (in the direction of signal flow).

A cone $C\left(s_{k}\right)$ is a function $s_{k}=f\left(s_{1}, s_{2}, \ldots, s_{p}\right)$ with a set of arguments $S_{k}=$ $\left\{s_{1}, s_{2}, \ldots, s_{p}\right\}=C\left(s_{k}\right)$ - $X$. It is a subnetwork $N\left(C\left(s_{k}\right)-X, s_{k}\right)$ with a set of inputs $C\left(s_{k}\right)_{-} X$, and with output $s_{k}$. A subcone $C^{\prime}\left(s_{k} \bar{\gamma}_{-}-C\left(s_{k}\right)\right.$ may have arguments which are not primary inputs. A gate is a smallest subcone.

Definition 2. Test patterns. For a circuit with $\mathrm{n}$ inputs, a test pattern $T_{l}$ is a $n$-bit ternary vector $T R^{n}, T R=\{0,1, u\}$, where $u$ - is a don't care. A set of test patterns $T=\left\{T_{1}\right\}$ is a test.

Definition 3. Stuck-at fault set. Let $F$ be the set of stuck-at-1(0) faults $\mathrm{s} / 1(0)$ for the variables $s \quad Z^{B} \quad X$ of the circuit. $F$ is a representative set of faults: to test all the stuck-at faults in the circuit, it is sufficient to test only 
the faults in $F$ [14]. On the other hand, when testing $F$, we test all the signal paths in all the tree-like subcircuits for transmitting both signals 1 and 0 .

Example: The representative set of faults in the circuit of Fig. 1 consists of 22 faults and is related to the variables: $\mathrm{s}_{1}, \mathrm{~s}_{2}, \mathrm{~s}_{3}, \mathrm{~s}_{4}, \mathrm{~s}_{5}, \mathrm{~s}_{6}, \mathrm{~s}_{7}, \mathrm{~s}_{3,1}, \mathrm{~s}_{3,2}, \mathrm{~s}_{8,1}, \mathrm{~s}_{8,2}$.

Note, the stuck-at fault model is not used here as a model for design error diagnosis. Its role is to provide only a measure for stating that one or another signal path is found to be erroneous. Detecting a fault in a path means that the path is going through an erroneous area (wrong gate, connection error etc.) in the circuit. We consider here the paths inside tree-like subcircuits of the design. There is a one-to-one correspondence between the paths and the variables $s_{-} Z^{B}{ }_{-} X$ used for defining $F$. A variable $s$ represents the path from the edge labelled by $s$ up to the closest edge labelled by $s_{k}\left(Z-Z^{B}\right){ }_{-} Y$. For example, $s_{3,1}$ represents the path through edges $s_{3,1}, s_{10}, s_{13}, s_{15}$. Detecting the fault $s_{3,1} / 0$ means that an error has been detected by testing this path for the value $s_{3,1}=1$. The information about faulty paths in the form of detected faults in $F$ is used later for carrying out diagnostic pre-analysis in order to identify as exact as possible the suspected erroneous areas in the circuit. The second advantage of using the stuck-at fault model is to have a possibility to use for design error diagnosis common ATPGs and fault simulators which have been developed initially for hardware testing purposes.

Consider a test $T=\left\{T_{1}\right\}$ that detects the representative set of faults $F=$ $\left\{\mathrm{s} / 0\right.$ and $\mathrm{s} / 1$ for $\left.s_{-} X_{-} Z^{B}\right\}$. This test may be obtained by any standard test generation technique for digital circuits [14]. The test is then simulated on the description of the implementation and on the specification. To improve the resolution of diagnostic pre-analysis or to detect the design errors not covered by the stuck-at fault test, additional test patterns may be needed.

Definition 4. Failed test patterns. If the result $y_{J}\left(T_{1}\right)_{-} w_{J}\left(T_{1}\right)$ is observed when applying the test pattern $T$, to the implementation and the specification, we say that the test pattern $T_{1}$ fails. Let $T^{*}-T$ be the subset of test patterns which fail during the design verification test.

Definition 5. Fault table. The fault table is a matrix _ $a_{l_{J}}$, where $\mathrm{a}_{\mathrm{i}, \mathrm{j}}=1$ (0) if the test pattern $T_{i}$ is able to detect the fault $s_{j} / 1(0)$, and is undetermined otherwise. Let $F_{i} \subset F$ be the set of faults which may be detected by $T_{l}$.

Definition 6. Suspected faults. A fault $\mathrm{s} / e, e_{-}\{0,1\}$, is called suspected if there is a pattern $T_{1} T^{*}$ which is able to detect this fault, and there exists no other pattern $T_{j}-\bar{T}-T^{*}$ which also is able to detect the same fault. Denote the whole set of suspected faults for the test $T$ at failed patterns $T^{*}$ by $F^{*}$.

Example: In Table 1, the first 7 rows form a set of 7 test patterns which cover all the representative faults of $F$ in Fig.1. In the left part of Table 1 the values of input and output variables for test patterns are given. In the right part detectable faults for test patterns are depicted. For example, the set of faults detectable by the pattern $T_{l}$ is $F_{l}=\left\{s_{l} / 0, s_{3} / 0, s_{3,2} / 0, s_{\downarrow} / 1, s_{5} / 1, s_{8,1} / 1\right\}$. 
Table 1. A test and its fault table for the circuit in Fig. 1 at given test

\begin{tabular}{|c|c|c|c|c|c|c|c|c|c|c|c|c|c|c|c|c|c|c|c|}
\hline \multirow{3}{*}{$\mathrm{i}$} & \multicolumn{8}{|c|}{ Test Patterns $\left(\mathrm{T}_{\mathrm{i}}\right)$} & \multicolumn{11}{|c|}{ Fault Table } \\
\hline & \multicolumn{7}{|c|}{ Inputs $\mathrm{j}: \mathrm{s}_{\mathrm{i}} \quad \mathrm{X}$} & \multirow{2}{*}{$\begin{array}{c}\text { Output } \\
\mathrm{s}_{15} \\
\end{array}$} & \multicolumn{11}{|c|}{$\mathrm{j}: \mathrm{s}_{\mathrm{i}} \mathrm{Z}^{\mathrm{B}} \quad \mathrm{X}$} \\
\hline & 1 & 2 & 3 & 4 & 5 & 6 & 7 & & 1 & 2 & $3_{1}$ & 3 & $33_{2}$ & 4 & 5 & 6 & 7 & $88_{1}$ & 8 \\
\hline 1 & 1 & 0 & 1 & 0 & 0 & 0 & 0 & 1 & 0 & & & 0 & 0 & 1 & 1 & & & 1 & \\
\hline 2 & 0 & 1 & 0 & 1 & 0 & 1 & 0 & 1 & & 0 & 1 & 0 & & 0 & & 0 & & & 0 \\
\hline 3 & 1 & 0 & 0 & 0 & 1 & 1 & 0 & 1 & 0 & & & & & & 0 & & 0 & & 0 \\
\hline 4 & 0 & 0 & 0 & 1 & 1 & 0 & 1 & 0 & & 1 & & & & & & & & & \\
\hline 5 & 0 & 1 & 1 & 0 & 0 & 0 & 1 & 0 & 1 & & 0 & & & & & & & & \\
\hline 6 & 1 & 0 & 0 & 0 & 0 & 1 & 0 & 0 & & & & 1 & 1 & 1 & 1 & & & & 1 \\
\hline 7 & 1 & 0 & 1 & 1 & 0 & 0 & 0 & 0 & & & & & & 0 & & 1 & 1 & 0 & \\
\hline 8 & 1 & 0 & 1 & 0 & 0 & 1 & 1 & 1 & 0 & & & 0 & 0 & 1 & 1 & & & 1 & \\
\hline
\end{tabular}

Definition 7. Error level of the variable. Let $F\left(s_{k}\right)-F$ be the set of possible faults in the cone $C\left(s_{k}\right)$, and $F^{*}\left(s_{k}\right)_{-} F\left(s_{k}\right)$ the set of suspected faults in $C\left(s_{k}\right) \cdot E\left(s_{k}\right)=F^{*}\left(s_{k}\right)_{-}{ }_{-} F\left(s_{k}\right) \_$is called error level of the variable $s_{k}$.

\section{GENERAL DESCRIPTION OF THE METHOD}

The procedure for error diagnosis and for circuit correction proposed in the present paper consists of the following steps.

1. Verification test with the goal to get a knowledge about faulty signal paths. This knowledge helps finding suspected erroneous areas in the circuit.

2. Diagnostic pre-analysis based on computing error levels $E(s)$ for all variables $s_{-} Y_{-}\left(Z-Z^{B}\right)$.

3. Defining a suspected erroneous subcircuit (subcone) $C^{\prime}(s)$.

4. Rectification of the function of the subcircuit $C^{\prime}(s)$ based on the results of the verification test, generating and executing new test patterns if needed.

5. If the rectification procedure corrects the design the problem is solved. Otherwise, steps 3 and 4 should be iteratively repeated.

In this procedure, for verification and for checking if the circuit is corrected by rectification (Step 4), the given set of test patterns $T$ is used. The test $\mathrm{T}$ may be extended by additional patterns either during Step 1 for increasing diagnostic resolution, or during Step 4. It may happen that Step 5 gives a positive result, although the verification with an equivalence prover still shows that the circuit rectified in Step 4 is not correct. When this happens, the test $\mathrm{T}$ should be extended by additional patterns.

\section{DIAGNOSTIC PRE-ANALYSIS}

The result of the verification test is a subset $T^{*}-T$ of failed test patterns. If no errors are found during verification test $\left(T^{*} \overline{-}_{-}\right)$, no diagnosis and no 
error correction should be made. On the basis of the test results and the fault table, the numbers of suspected faults $\left|F^{*}(s)\right|$ and error levels $E(s)$ for all variables $s_{-} Y_{-}\left(Z-Z^{B}\right)$ are computed by the following algorithm.

Algorithm 1.

1. From the list $T^{*}$ of failed test patterns, the preliminary set of suspected faults is constructed: $\mathrm{F}^{1}=\cup_{\mathrm{Ti}_{-} \mathrm{T}^{*}} \mathrm{~F}_{\mathrm{i}}$.

2. From the list $T-T^{*}$ of passed test patterns, the set of faults which definitely cannot be present is calculated: $\mathrm{F}^{2}=\cup_{\mathrm{Ti}_{-} \mathrm{T}-\mathrm{T}^{*}} \mathrm{~F}\left(\mathrm{~T}_{\mathrm{i}}\right)$.

3. The subset of suspected faults is now calculated as: $F^{*}=F^{l}-F^{2}$.

4. The subsets of suspected faults observable (detectable) at edges $s_{-} Y$ $\cup\left(Z-Z^{B}\right)$ are calculated as: $F^{*}(s)=F(s) \_F^{*}$.

5. Error levels for $s_{-} Y_{-}\left(Z-Z^{B}\right)$ are calculated: $E(s)=F^{*}(s){ }_{-}{ }_{-} F(s)$.

Example: Consider the circuit in Fig. 1 with two design errors. The test given in Table 1 is good for error detection, as it covers all the possible stuck-at faults. On the other hand, the diagnostic resolution for the given test is very low. The failure of patterns $T_{1}, T_{2}$, and $T_{3}$ and the non-failure of the other patterns implies that all the representative edges (paths) of the circuit except $s_{4}$ remain suspected as faulty. The set of suspected faults is $F^{*}=$ $\left\{s_{1} / 0, s_{2} / 0, s_{3,1} / 1, s_{3,2} / 0, s_{5} / 0, s_{6} / 0, s_{7} / 0, s_{8,1} / 1, s_{8,2} / 0\right\}$. Such information helps very little for locating the erroneous area in the circuit. Adding an additional pattern $T_{8}=(1010011)$ that does not fail, significally improves the diagnostic resolution. The set of suspected faults now consists of 6 faults: $F^{*}=\left\{s_{2} / 0\right.$, $\left.s_{3,1} / 1, s_{5} / 0, s_{6} / 0, s_{7} / 0, s_{8,2} / 0\right\}$ (located at bold lines in Fig.1). The results of calculating error levels for the circuit are shown in Table 2.

Table 2. Error levels for variables of the circuit in Fig.1 for the given test experiment

\begin{tabular}{|l|c|c|c|c|c|c|c|c|}
\hline Variables (s) & $\mathrm{s}_{8}$ & $\mathrm{~s}_{9}$ & $\mathrm{~s}_{10}$ & $\mathrm{~s}_{11}$ & $\mathrm{~s}_{12}$ & $\mathrm{~s}_{13}$ & $\mathrm{~s}_{14}$ & $\mathrm{~s}_{15}$ \\
\hline Number of faults in the cone C(s) & 4 & 4 & 4 & 8 & 10 & 6 & 14 & 22 \\
\hline Number of susp. faults in C(s) & 1 & 2 & 2 & 1 & 4 & 2 & 4 & 6 \\
\hline Error level of the variable E(s) & 0,25 & 0,5 & 0,5 & 0,13 & 0,4 & 0,33 & 0,29 & 0,27 \\
\hline
\end{tabular}

On the basis of the results of Algorithm 1, the suspected erroneous area may now be predicted. This is done by two procedures: elimination of pseudofaults, and suppressing the faulty area by using error level function.

\subsection{Elimination of not distinguishable pseudofaults}

It is rather realistic to suppose that there is in $F^{*}$ a subset of suspected faults $F^{\prime} \subset F^{*}$ which belongs to the paths not crossing the actual erroneous area in the circuit. Let us call these suspected faults in $F^{\prime}$ as pseudofaults. The existence of pseudofaults in $F^{*}$ may mislead us in making decisions about suspected erroneous areas. The reason of existing of pseudofaults is 
the absence of a nonfailing test pattern in $T$ which would be able to distinguish the faults in $F^{\prime}$ from the faults in $F^{*}$, i.e. which would be able to detect a fault in $F^{\prime}$, and not to detect faults in $F^{*}-F^{\prime}$.

Consider the circuit in Fig. 2 with an erroneous gate $O R_{5}$ (instead of $O R_{5}$ there should be AND gate). The test $T_{l}=110$ will fail because of the erroneous $O R_{5}$. On the other hand, $T_{l}$ detects faults $s_{l} / 0$ and $s_{2} / 0$, and there is no other test pattern which can distinguish these faults. Hence, from failing of the test $T_{1}$ we have to conclude that both $O R_{4}$ and $O R_{5}$ may be erroneous.

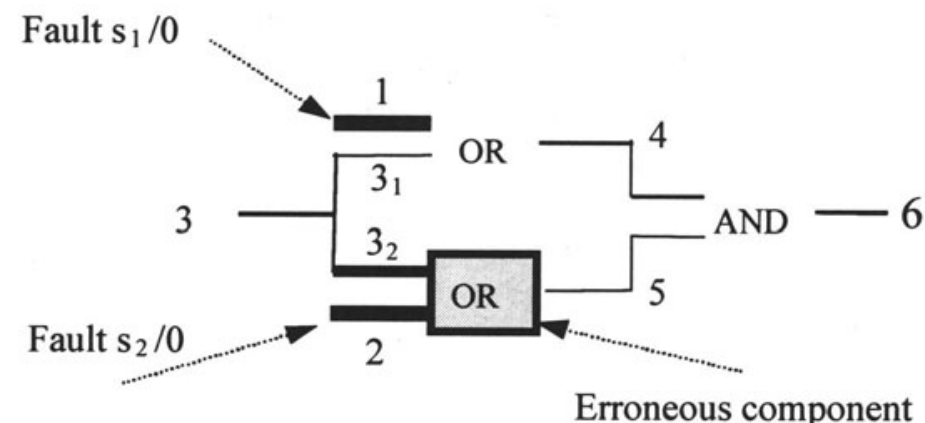

Figure 2. Diagnosis with bad resolution (faults $s_{1} / 0$ and $s_{2} / 0$ are not distinguishable

Consider now the full test for the circuit. The set of failed test patterns will be $T^{*}=\{001,101,110\}$ which gives a set of suspected faults $F^{*}=$ $\left\{\mathrm{s}_{1} / 0, \mathrm{~s}_{2} / 0, \mathrm{~s}_{3,2} / 0\right\}$. Only the cone $C\left(s_{6}\right)$ covers all the faults in $F^{*}$, which leads to the conclusion that rectification should be carried out for the whole circuit. This would be needed for a multiple error when both $C\left(s_{+}\right)$and $C\left(s_{5}\right)$ were erroneous. Considering that the probability of multiple errors is low, we assume that only one of the cones $C\left(s_{4}\right)$ and $C\left(s_{5}\right)$ can be erroneous, and that the other one contains pseudofaults. It is also reasonable to expect that the number of pseudofaults is always less than the number of other suspected faults in $F^{*}$. This results from the expectation that not all suspected faults in the erroneous area have a counterpart in $F^{\prime}$. Here, $F^{\prime}=F^{*}\left(s_{+}\right)$contains the pseudofault $s_{1} / 0$ which is not distinguishable from $s_{2} / 0$ in $F^{*}\left(s_{5}\right)$. However, $s_{3,2} / 0$ in $F^{*}\left(s_{5}\right)$ has no distinguishable counterpart in $F^{*}\left(s_{+}\right)$.

Rule 1. If two subcones $C\left(s_{1}\right)$ and $C\left(s_{j}\right)$ with $F^{*}\left(s_{l}\right)$ and $F^{*}\left(s_{\nu}\right)$ are feeding the same component, and if $\left|C\left(s_{l}\right)\right|>\left|C\left(s_{\nu}\right)\right|$ then only the cone $C\left(s_{1}\right)$ should be suspected as erroneous, and the redesign should start with this cone.

All the cones qualified to contain pseudofaults like $C\left(s_{\nu}\right)$, are put into STACK for possible backtracking in the case of wrong predictions.

Example: In Fig. 1 only the full cone $C\left(s_{15}\right)$ covers all suspected faults. However, for the inputs of $A N D_{15}$ we have: $\left|C\left(s_{13}\right)\right|=2$, and $\left|C\left(s_{1+4}\right)\right|=4$, which along Rule 1 makes us to predict that the faults in $C\left(s_{13}\right)$ are pseudo. Further, for the inputs of $O R_{14}$ we have: $\left|C\left(s_{11}\right)\right|=1$, and $\left|C\left(s_{12}\right)\right|=4$. However, the fault in $C\left(s_{11}\right)$ contains also in $C\left(s_{12}\right)$, and therefore, the Rule 1 is not usable. 


\subsection{Diagnosis by error level function.}

After choosing by Rule 1 the cone $C(s)$ suspected to be erroneous, we may try to suppress even more the suspected area, instead of rectifying the whole network defined by $C(s)$. As the error level $E(s)$ refers to the number of suspected faults in relation to the whole number of faults in $C(s)$, we are justified to expect that the higher the error level of $s$, the higher is the probability that $C(s)$ is erroneous. This leads to the second rule.

Rule 2. Among all the possible cones in $C(s)$ chosen by Rule 1 , a cone $C\left(s_{k}\right)$ where $E\left(s_{k}\right)=$ max over all $s_{t}\{C(s)-X, s\}$ should be rectified.

Assuming again that the probability of multiple design errors is less than the probability of single errors, we may expect that all the faulty paths in the suspected cone $C\left(s_{k}\right)$ are caused by an error related to the top component of the cone. This leads to the third rule for choosing the target for redesign.

$\underline{\text { Rule } 3}$. In the cone $C\left(s_{k}\right)$ chosen by Rule 2 , at first, its top component (the smallest subcone $C^{\prime}\left(s_{k}\right)$ in $C\left(s_{k}\right)$ ) should be taken for rectification.

If the rectification of $C^{\prime}\left(s_{k}\right)$ does not correct the circuit, we have to either increase the suspected area in $C\left(s_{k}\right)$ towards inputs or to choose another cone from $C(s)$ (or from STACK). The decision is made again on the basis of error level values. The procedure for correcting the circuit is as follows.

\section{Algorithm 2.}

1. Select by Rule 1 a cone $C(s)$, and create STACK of eliminated cones.

2. Select by Rule 2 a cone $C\left(s_{k}\right)$ in $C(s)$.

3. Select by Rule 3 a subcone $C^{\prime}\left(s_{k}\right)$ for redesigning to correct the error.

4. If redesign explains the error, algorithm is ended. Otherwise, go to 8 .

5. Choose the variable $s_{l} \in X$ with $E\left(s_{l}\right)=$ max over all the inputs of $C^{\prime}\left(s_{k}\right)$ and over all the variables in $C(s)$ outside $C^{\prime}\left(s_{k}\right)$.

6. If $s_{1}$ is an input of $C^{\prime}\left(s_{k}\right)$, update the subcone $C^{\prime}\left(s_{k}\right)$ by including the top component of the cone $C\left(s_{l}\right)$ into it. Go to 4.

7. If $\mathrm{s}_{l}$ is a variable outside $C^{\prime}\left(s_{k}\right)$, take $s_{k}=s_{l}$. Go to 3 .

8. If $C^{\prime}\left(s_{k}\right)=C(s)$, take $C(s)$ from STACK, and go to 2 . Otherwise, go to 5 .

Example: According to Step 1 in Algorithm 2 (see previous Example), we eliminate $C\left(s_{13}\right)$, and accept $C\left(s_{14}\right)$ as suspected erroneous area. Steps 2,3 suggest to restrict this area to the cone $C\left(s_{9}\right)=O R_{9}$ where $E\left(s_{9}\right)=0,5=\max$. However, in Step 4 we find that redesign of $O R_{9}$ cannot explain the error. Steps 5,7,3 suggest now to try with $A N D_{12}$ in $C\left(s_{12}\right)$ where $E\left(s_{12}\right)=0,4=$ max. The next choice will be $C^{\prime}\left(s_{9}\right)=O R_{9}$, where $E\left(s_{9}\right)=0,5$, and, according to Step 6 , we try to explain the design error by redesigning the network of $O R_{9}$ and $A N D_{12}$. After the rectification again fails, we try with $C^{\prime}\left(s_{14}\right)=O R_{14}$, where $E\left(s_{14}\right)=0,29$, and then include to this subcone the gate $C^{\prime}\left(s_{12}\right)=A N D_{12}$ since $E\left(s_{12}\right)>E\left(s_{11}\right)$. The last attempt to correct the circuit by redesigning the network of $O R_{14}$ and $A N D_{12}$ has success (see Example in Section 5) . 


\section{RECTIFICATION OF THE CIRCUIT}

For rectifying the function of a subnetwork $N\left(S_{k}, s_{k}\right)$ with output $s_{k}$ and input set $S_{k}$, we have to choose test patterns from the initial test set $T$ (or create new patterns if the needed ones are missing in $T$ ), to put together a new set of patterns $T^{\prime}$ 'so that $T^{*} \subseteq T^{\prime}$, and which includes all possible value combinations of the variables $s_{-} S_{k}$, so that for each $T_{l_{-}} T^{\prime}$ ' at least for one $y_{j} \_Y$ the following holds: $y_{J} / s_{k}=1$. The last condition makes the variable $s_{k}$ observable at the output(s) and the behaviour of the subnetwork $N\left(S_{k}, s_{k}\right)$ can be corrected, if $y_{j}\left(T_{i}\right) \_w_{j}\left(T_{i}\right)$ is observed. The number of patterns in $T$, is $\left|T^{\prime}\right| \geq 2^{p}$, where $p$ is the number of variables in $S_{k}$. Correction of the chosen subnetwork $N\left(S_{k}, s_{k}\right)$ means that when extracting the behaviour of $s_{k}$ from the simulation results of $T^{\prime}$, the value of $s_{k}$ should be changed for all the failed test patterns in $T^{\prime}$. For the new function derived in such a way, a new network $N^{\prime}\left(S_{k}, s_{k}\right)$ is designed.

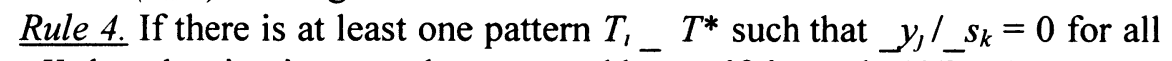
$y_{J}-Y$, then the circuit cannot be corrected by rectifying only $N\left(\bar{S}_{k}, s_{k}\right)$.

Indeed, because of $y_{J} / s_{k}=0$ for all $y_{J} Y$, the cause of failing $T_{1}$ should be somewhere else than the changed value of $s_{k}$.

For the circuit with new $N^{\prime}\left(S_{k}, s_{k}\right)$, test verification experiment is repeated with $T^{\prime}$. If all the patterns in $T^{\prime}$ pass, the new design is correct in relation to the verification test. If at least one pattern from $T$ ' fails, the rectification procedure is repeated with another suspected erroneous area.

Example: In the previous example, corresponding to Algorithm 2 we appointed for rectification several subnetworks in the following order: $O R_{g}$, $A N D_{12}, A N D_{12}+O R_{9}, O R_{14}$, and $O R_{14}+A N D_{12}$. In the following the rectification trials are shown for $A N D_{12}, O R_{14}$, and $O R_{14}+A N D_{12}$. The results of rectifying $A N D_{12}$ are given in Table 3 in the column $s_{12}=f\left(s_{8}, s_{9}\right)$ where $T^{\prime}=\left\{T_{1}, T_{2}, T_{3}, T_{6}, T_{7}\right\}$. The results of testing are given in the column Y/W. Already the first pattern $T_{1}$ shows that along Rule 4, by rectifying $A N D_{12}$ the circuit cannot be corrected: for $T_{1}$ we find that $y_{15} / s_{12}=0$. Hence, the error in $A N D_{12}$ cannot explain the erroneous behaviour of the circuit. The results of rectifying $O R_{14}$ are given in the column $s_{14}=f\left(s_{11}, s_{12}\right)$. As the pattern $\left(s_{11}=s_{12}=1\right)$ is not possible we can substitute this function by constant 0 . However, the repeated simulation of the test $T$ shows that the circuit is still erroneous. By extending now the suspected area towards inputs, we try to rectify the network $O R_{14}+A N D_{12}$ with function $s_{14}=$ $f\left(s_{8}, s_{9,}, s_{11}\right)$. The results are in the last column. To create a test $T$, we have to generate new patterns $T_{10}, T_{11}, T_{12}$ to get the full set of possible values at the inputs of the network under redesign, and to satisfy the condition $y_{15} / s_{14}=$ 1. It comes out that two input combinations are inconsistent for the given circuit, which means that $s_{1+}=u$ (don't care) for the inputs 101 and 111 . 
After substituting $u=0$, we find the rectified function as: $s_{1+}=s_{3} \& \_s_{\gamma} \& s_{9}$. It is easy to see that this is equivalent to $s_{1+}=s_{1 /} \&\left(s_{8} s_{g}\right)$. The circuit is corrected.

Table 3. Rectification of suspected erroneous functions

\begin{tabular}{|c|c|c|c|c|c|c|c|c|c|}
\hline \multirow{3}{*}{ No } & \multicolumn{3}{|c|}{ Test patterns } & \multicolumn{6}{|c|}{ Rectification actions } \\
\hline & \multirow{2}{*}{$\begin{array}{c}\text { Inputs } \\
1234567 \\
\end{array}$} & Internal signals & \multirow{2}{*}{$\frac{\mathrm{Y} / \mathrm{W}}{15}$} & \multicolumn{2}{|c|}{$\mathrm{s}_{12}=\mathrm{f}\left(\mathrm{s}_{8}, \mathrm{~s}_{9}\right)$} & \multicolumn{2}{|c|}{$\mathrm{s}_{14}=\mathrm{f}\left(\mathrm{s}_{11}, \mathrm{~s}_{12}\right)$} & \multicolumn{2}{|c|}{$\mathbf{s}_{14}=\mathbf{f}\left(\mathbf{s}_{8}, \mathbf{s}_{9}, \mathbf{s}_{11}\right)$} \\
\hline & & $\begin{array}{l}8 \quad 911121314 \\
\end{array}$ & & 89 & 12 & 1112 & 14 & 8911 & 14 \\
\hline 1 & 1010000 & $\begin{array}{llllll}0 & 0 & 1 & 0 & 1 & 1\end{array}$ & $1 / 0$ & & - & 10 & 0 & $\begin{array}{lll}0 & 0 & 1\end{array}$ & 0 \\
\hline 2 & 0101010 & $\begin{array}{llllll}1 & 1 & 0 & 1 & 1 & 1\end{array}$ & $1 / 0$ & & 0 & & 0 & 110 & 0 \\
\hline 3 & 1000010 & $\begin{array}{llllll}1 & 1 & 0 & 1 & 1 & 1\end{array}$ & $1 / 0$ & & 0 & & 0 & 110 & 0 \\
\hline 4 & 00001101 & $\begin{array}{llllll}1 & 1 & 0 & 1 & 0 & 1\end{array}$ & $0 / 0$ & 11 & 0 & & & & \\
\hline 5 & 0110001 & $\begin{array}{llllll}0 & 1 & 1 & 0 & 0 & 1\end{array}$ & $0 / 0$ & & & & & & \\
\hline 6 & 1000010 & $\begin{array}{llllll}0 & 1 & 0 & 0 & 1 & 0\end{array}$ & $0 / 0$ & $\begin{array}{ll}0 & 1\end{array}$ & 0 & $\begin{array}{ll}0 & 0\end{array}$ & 0 & $\begin{array}{lll}0 & 1 & 0\end{array}$ & 0 \\
\hline 7 & 1011000 & $\begin{array}{llllll}1 & 0 & 0 & 0 & 1 & 0 \\
\end{array}$ & $0 / 0$ & 10 & 0 & $\begin{array}{ll}0 & 0 \\
\end{array}$ & 0 & $\begin{array}{lll}1 & 0 & 0 \\
\end{array}$ & 0 \\
\hline 8 & 10010011 & $\begin{array}{llllll}0 & 1 & 1 & 0 & 1 & 1 \\
\end{array}$ & $1 / 1$ & & & & & $\begin{array}{lll}0 & 1 & 1 \\
\end{array}$ & 1 \\
\hline 9 & Not possible & & & & & 11 & - & & \\
\hline 10 & 1000000 & $\begin{array}{llllll}0 & 0 & 0 & 0 & 1 & 0\end{array}$ & $0 / 0$ & & & & & $\begin{array}{lll}0 & 0 & 0\end{array}$ & 0 \\
\hline 11 & Not possible & & & & & & & $\begin{array}{lll}1 & 0 & 1\end{array}$ & - \\
\hline 12 & Not possible & & & & & & & $\begin{array}{lll}1 & 1 & 1\end{array}$ & - \\
\hline
\end{tabular}

\section{EXPERIMENTAL DATA}

The main goal of the experiments was to evaluate the design error diagnostic properties of test patterns generated by traditional ATPGs for only stuck-at fault detecting purposes. The computer platform was: Sun SparcServer 20 (2 SuperSparc II microprocessors, $75 \mathrm{MHz}$ ), Solaris 2.5.1. operating system.

In the experiments, ISCAS' 85 benchmarks were used (columns 1,2,3,4 in Table 4). Test patterns for detecting stuck-at faults were created by the test generator Turbo Tester described in [15]. Fault coverage (6) and test generation time in seconds (11) are presented in Table 4. For all circuits, randomly selected single gate errors were inserted. The numbers of experiments carried out for each benchmark circuit are shown in the column 5. The total diagnosis time (13) consists of: test generation time (11) and fault diagnosis time (12). The time for rectification is not taken into account in these experiments.

The columns 7-9 show, correspondingly, the minimum, maximum and average diagnostic resolutions (numbers of suspected gates) reached by the test patterns generated only for fault detecting purposes and not for fault localization. The numbers in the column 10 illustrate the average sizes of the suspected erroneous areas in the circuits for the given test patterns. 
Table 4. Experimental data about design error diagnosis with ISCAS' 85 benchmarks

\begin{tabular}{|c|c|c|c|c|c|c|c|c|c|c|c|c|}
\hline \multirow[t]{3}{*}{ Circuit } & \multicolumn{4}{|c|}{ Number of } & \multirow{3}{*}{$\begin{array}{c}\text { Fault } \\
\text { cover } \\
\%\end{array}$} & \multicolumn{4}{|c|}{ Suspected gates } & \multicolumn{3}{|c|}{ Time, $s$} \\
\hline & \multirow[t]{2}{*}{ inputs } & \multirow[t]{2}{*}{ outputs } & \multirow[t]{2}{*}{ gates } & \multirow{2}{*}{$\begin{array}{l}\text { experi- } \\
\text { ments }\end{array}$} & & \multirow[b]{2}{*}{ Min } & \multirow[b]{2}{*}{ Max } & \multirow[b]{2}{*}{ Av. } & \multirow{2}{*}{$\begin{array}{c}\text { Av. } \\
\%\end{array}$} & \multirow{2}{*}{\begin{tabular}{|l} 
Test \\
gener.
\end{tabular}} & \multirow{2}{*}{\begin{tabular}{|l} 
Fault \\
analysis
\end{tabular}} & \multirow[t]{2}{*}{ Total } \\
\hline & & & & & & & & & & & & \\
\hline 1 & 2 & 3 & 4 & 5 & 6 & 7 & 8 & 9 & 10 & 11 & 12 & 13 \\
\hline c432 & 36 & 7 & 232 & 116 & 91,07 & 1 & 107 & 9 & 3,8 & 0,8 & 0,1 & 0,9 \\
\hline c499 & 41 & 32 & 618 & 106 & 99,33 & 1 & 307 & 77 & 12,3 & 1,0 & 1,4 & 2,4 \\
\hline c880 & 60 & 26 & 357 & 177 & 100 & 1 & 33 & 6 & 1,7 & 0,19 & 0,5 & 0,69 \\
\hline $\mathrm{c} 1355$ & 41 & 32 & 514 & 418 & 99,51 & 1 & 248 & 58 & 11,3 & 1,35 & 1,5 & 2,85 \\
\hline c1908 & 33 & 25 & 718 & 378 & 99,31 & 1 & 76 & 11 & 1,6 & 0,93 & 1,6 & 2,53 \\
\hline c2670 & 233 & 140 & 997 & 343 & 94,97 & 1 & 161 & 25 & 2,6 & 3,55 & 14,1 & 17,7 \\
\hline c3540 & 50 & 22 & 1446 & 458 & 95,27 & 1 & 86 & 10 & 0,7 & 3,08 & 3,7 & 6,78 \\
\hline c5315 & 178 & 123 & 1994 & 695 & 98,69 & 1 & 239 & 1 & 0,6 & 2,38 & 29,4 & 31,8 \\
\hline c6288 & 32 & 32 & 2416 & 2128 & 99,34 & 1 & 138 & 8 & 0,4 & 2,17 & 2,7 & 4,87 \\
\hline c7552 & 207 & 108 & 2978 & 1326 & 95,95 & 1 & 269 & 6 & 0,5 & 12,06 & 44,8 & 79,6 \\
\hline
\end{tabular}

\section{CONCLUSIONS}

In this paper, a new approach to design error diagnosis in combinational circuits without error model is proposed. The procedure combines a diagnostic pre-analysis for predicting the suspected erroneous area, and resynthesis for correcting the design. For representing the information about erroneous signal paths in the circuit, a stuck-at fault model is used. This allows to adopt the methods and tools of fault diagnosis used in hardware testing [14] for the use in design error diagnostic pre-analysis.

Differently from the known design error diagnosis methods which apply the re-design technique for randomly chosen cones of the circuit, in our paper, diagnostic pre-analysis is used to systematically compress the suspected erroneous area. Contrary to other published works, here the necessary re-synthesis of the extracted subcircuit need not be applied to the whole function of an internal signal in terms of primary inputs, but may stop at arbitrary nodes inside the circuit to rectify embedded subfunctions.

Our approach has two advantages compared to other re-design based methods: we use diagnostic pre-analysis to concentrate the rectification to small suspected erroneous areas, and by rectifying embedded subfunctions we can avoid the combinatorial explosion of OBDD based methods. The size of the circuits to be rectified depends on the quality of diagnostic preanalysis. The method can be applied also to sequential circuits if scan-path technique is used.

The shortcomings of the proposed method are the lack of exact deterministic technique for diagnostic pre-analysis. Some heuristics is used 
for selecting the suspected erroneous areas; this is rather natural, because a very general case is considered: (1) all errors including multiple ones are allowed, and therefore no error model is used; (2) no structural similarity is assumed between the specification and the implementation.

Future work will be devoted to the development of deterministic approaches for more exact prediction of the erroneous area and to the development of methods for generating diagnostic test patterns with better resolution.

\section{Acknowledgement}

The authors appreciate the work of Jaan Raik and Artur Jutman from Tallinn Technical University for carrying out the experiments. They thank also the reviewers for valuable comments and suggestions.

\section{REFERENCES}

[1] A.M. Wahba, D. Borrione. Connection Error Location in Combinational Circuits. Proc. European Design \& Test Conference, Paris, March 17-20, 1997, pp.235-241.

[2] P.Y. Chung, Y.M. Wang, I.N. Hajj. Diagnosis and Correction of Logic Design Errors in Digital Circuits. ACM/IEEE Design Automation Conference, pp. 503-508, 1993.

[3] I. Pomeranz, S.M. Reddy. On Error Correcting in Macro-based Circuits. Proceedings. of ICCAD, pp.568-575, 1994.

[4] A.M. Wahba, D. Borrione. A Method for Automatic Design Error Location and Correction in Combinational Logic Circuits. J. of Electronic Testing: Theory and Applications 8, 1996, pp. 113-127.

[5] R. Ubar, D. Borrione. Single Gate Design Error Diagnosis in Combinational Circuits. Proc. Estonian Acad. Sci. Engng., 1999, 5, 1, 3-21.

[6] D. Brand, A. Drumm, S. Kundu, P. Narrain. Incremental Synthesis. Proceedings of ICCAD, pp.14-18, 1994.

[7] S.-Y. Huang, K.-C. Chen, K.-T. Cheng. Error Correction based on Verification Techniques. ACM/IEEE Design Automation Conference, pp. 258-261, 1996.

[8] S.-Y. Huang, K.-C. Chen, K.-T. Cheng. Incremental Logic Rectification. Proc. of VLSI Test Symposium, 1997, pp. 134-139.

[9] J.C. Madre, O.Coudert, J.P. Billon. Automating the Diagnosis and the Rectification of the Design Errors with PRIAM. Proceedings of ICCAD, pp. 30-33, 1989.

[10] C.-C. Lin, K.-C. Chen, S.-C. Chang, M. Marek-Sadowska, K.-T. Cheng. Logic Synthesis for Engineering Change. ACM/IEEE DAC, pp. 647-652, 1995.

[11] D.W. Hoffmann, T. Kropf. Automatic Error Correction of Large Circuits Using Boolean Decomposition and Abstraction. Proc. of CHARME, to be published.

[12] U. Watanabe, R.K. Brayton. Incremental Synthesis for Engineering Change. Proceedings of ICCAD, pp. 40-43, 1991.

[13] R.E. Bryant. Graph-based Algorithm for Boolean Function Manipulation. Proceedings of ICCAD, pp. 316-319, 1989.

[14] M. Abramovici, M.A. Breuer, A.D. Friedman. Digital Systems Testing and Testable Designs. 1998.

[15] J.Raik, R.Ubar. Feasibility of Structurally Synthesized BDD Models for Test Generation. Proc. of the IEEE European Test Workshop. Barcelona, May 27-29, 1998, pp.145-146. 